\title{
Prediction of pathologic upgrading in Gleason score $3+4$ prostate cancer: Who is a candidate for active surveillance?
}

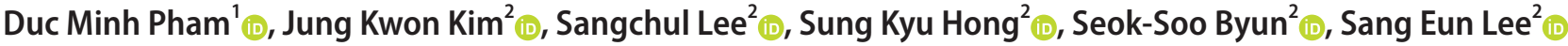 \\ ${ }^{1}$ Department of Urology, Cho Ray Hospital, Ho Chi Minh, Viet Nam, ${ }^{2}$ Department of Urology, Seoul National University Bundang Hospital, Seongnam, Korea
}

Purpose: Whether active surveillance (AS) can be safely extended to patients with Gleason score (GS) 3+4 prostate cancer is highly debated. We examined the incidence and predictors of upgrading among patients with GS 3+4 disease.

Materials and Methods: The study involved 377 patients with biopsy GS 3+4 who underwent robot-assisted laparoscopic radical prostatectomy (RP) from 2014 to 2018 at a single institution. We analyzed the rate of GS upgrading and used logistic regression to determine the predictors of upgrading.

Results: A total of 168 (44.6\%) patients with GS 3+4 experienced an upgrade in GS. In multivariable analysis, advanced age, prostate-specific antigen (PSA) level, PSA density (PSAD) and Prostate Imaging-Reporting and Data System version 2 (PI-RADS v2) score were significant predictors of GS upgrading. When structured into a predictive model that included age $\geq 65$ years, PSA $\geq 7.7$ $\mathrm{ng} / \mathrm{mL}$, PSAD $\geq 0.475 \mathrm{ng} / \mathrm{mL}^{2}$ and PI-RADS v2 score $4-5$, the probability of GS upgrading ranged from $36.4 \%$ to $65.7 \%$ when one to four of these factors were included.

Conclusions: A substantial proportion of patients with GS 3+4 prostate cancer were upgraded after RP. However, according to our model combining clinical and imaging predictors, patients with a low risk of GS upgrading may be eligible candidates for AS.

Keywords: Pathology; Prognosis; Prostate neoplasms; Prostatectomy

This is an Open Access article distributed under the terms of the Creative Commons Attribution Non-Commercial License (http://creativecommons.org/licenses/by-nc/4.0) which permits unrestricted non-commercial use, distribution, and reproduction in any medium, provided the original work is properly cited.

\section{INTRODUCTION}

Prostate cancer $(\mathrm{PCa})$ has the highest incidence rate and the second-highest mortality rate of the common cancers in males [1]. However, many newly diagnosed PCa patients present in early stage when the disease is indolent or nonprogressing and is unlikely to lead to morbidity or mortality [2]. For this reason, active surveillance (AS) has emerged as an attractive option for low-risk PCa to avoid overtreatment and reserve the opportunity for definitive therapy if the disease progresses. Whether or not AS can be safely extended to patients with Gleason 3+4 PCa remains a highly debated issue. Some data have shown that intermediate-risk (IR) PCa has similar oncologic outcomes to low-risk disease following curative treatment [3-5], suggesting that some Gleason score (GS) 3+4 patients may be candidates for AS. The National Comprehensive Cancer Network (NCCN) guidelines list AS an option for males with favorable IR PCa (GS 3+4, <50\%

Received: 17 December, 2019 - Accepted: 17 February, 2020

Corresponding Author: Sang Eun Lee (iD https://orcid.org/0000-0001-5480-9970

Department of Urology, Seoul National University Bundang Hospital, 82 Gumi-ro 173beon-gil, Bundang-gu, Seongnam 13620, Korea

TEL: +82-31-787-7341, FAX: +82-31-787-4057, E-mail: selee@snubh.org 
positive biopsy cores and one additional IR factor) [6], while the America Society of Clinical Oncology endorses AS for select patients with low-volume Gleason 3+4 disease [7]. However, criteria identifying "favorable" IR have not yet been defined clearly due to lack of supporting oncologic evidence.

Although the GS score is regarded as the single most important determinant in risk assessment, evidence supporting AS in the majority of studies is based on biopsy results instead of whole-specimen pathology [8,9]. Many patients with GS 3+4 prostate cancer harboring higher grade disease than their biopsy need immediate active treatment, rather than AS, because of the high potential to progress [10]. We, therefore, examined the incidence and predictors of upgrading in GS 3+4 patients underwent radical prostatectomy (RP) to provide simple guidance on selecting GS $3+4$ patients who would benefit from AS.

\section{MATERIALS AND METHODS}

This retrospective study was approved by Institutional Review Board of Seoul National University Bundang Hospital (IRB number: B-1706/402-115). We identified 1,791 males who underwent robot-assisted laparoscopic RP from 2014 to 2018. Transrectal ultrasound-guided prostate biopsy and multiparametric magnetic resonance imaging (mpMRI) were performed preoperatively on all of the patients according to the Prostate Imaging-Reporting and Data System version 2 (PI-RADS v2) guidelines. After excluding patients who had incomplete biopsy data $(n=25)$ and those who had not assessed PI-RADS v2 classification in mpMRI ( $\mathrm{n}=516), 1,250$ patients with various GS score were examined to identify patients with GS 3+4 PCa. Eventually, a database of 377 patients was analyzed in our study.

The clinical and biopsy variables included age, prostate volume, prostate-specific antigen (PSA) level, PSA density (PSAD), number of positive cores, maximum tumor length in a core, PI-RADS v2 score, number of lesions in mpMRI and clinical tumor stage. Pathologic variables were primary and secondary GS on biopsy samples and surgical specimens. GS was assigned according to the 2005 International Society of Urological Pathology (ISUP) modified Gleason scoring system. The primary outcome was GS upgrading, defined as an increase to GS $\geq 4+3$ after RP.

The patients were divided into the upgraded and nonupgraded groups. We used chi-squared tests to compare the categorical variables and independent t-tests or MannWhitney test to compare the continuous variables. Univariable and multivariable logistic regression analyses were applied to determine the predictors of GS upgrading. All tests were two-sided with significance assumed at $p<0.05$. Statistical analyses were performed using IBM SPSS ver. 22.0 (IBM Corp., Armonk, NY, USA).

\section{RESULTS}

The baseline demographic and clinical features of 377 patients who were diagnosed with GS 3+4 $\mathrm{PCa}$ and underwent RP are shown in Table 1 . Among the 377 total patients, the GS was upgraded in 168 (44.6\%) after RP. Of these upgraded, 155 (92:3\%) were upgraded to GS $4+3$, four (2.4\%) to GS 4+4, and nine (5.3\%) to GS 4+5. In 209 subjects whose GS were not upgraded, only $1(0.5 \%)$ was downgraded to GS $3+3$ and 208 (99.5\%) remained GS 3+4. Comparison of the nonupgraded and upgraded patients demonstrated that there was no statistical difference in prostate volume, number of positive cores, percentage of positive cores, maximum tumor length in a core, maximum percentage of tumor length in a positive core in biopsy samples, number of lesions in mpMRI and clinical tumor stages. In contrast, the upgraded group showed significantly higher age, preoperative PSA level, PSAD and PI-RADS v2 score.

Using receiver operating characteristic curve analysis, we identified the optimal cut-off value for continuous variables associated with GS upgrading, including age, PSA, and $\mathrm{PSAD}$ in order to change them into categorical variables for easily clinical application. Using univariate logistic regression analysis to determine the predictors, the results indicated that GS upgrading was positively associated with age $\geq 65$ ( $p=0.032$ ), PSA level $\geq 7.7 \mathrm{ng} / \mathrm{mL}(\mathrm{p}<0.001)$, PSAD $\geq 0.475$ $\mathrm{ng} / \mathrm{mL}^{2}(\mathrm{p}<0.001)$ and PI-RADS v2 score $4-5(\mathrm{p}=0.007)$. In a further multivariate logistic regression model using these factors, all four elements were significantly related to GS upgrading (Table 2).

The GS upgrading predictive model was structured to stratify patients according to four risk factors: age $\geq 65$, PSA level $\geq 7.7 \mathrm{ng} / \mathrm{mL}$, PSAD $\geq 0.475 \mathrm{ng} / \mathrm{mL}^{2}$, and PI-RADS v2 score $4-5$. The rate of GS upgrading in patients with GS $3+4 \mathrm{PCa}$ is shown in Table 3 and ranged from $36.4 \%$ when at least one of four predictive factors was included to $65.7 \%$ when all four factors were present $(p<0.001)$.

\section{DISCUSSION}

Although AS is an alternative management strategy for patients who have mainly low-risk GS $3+3$ cancer, several AS protocols include GS $3+4$ PCa patients $[11,12]$. Males with GS $3+4$ on biopsy were comparable to those with GS $3+4$ in terms of biochemical recurrence and mortality [3,4]. 
Table 1. Patient demographics and clinicopathologic characteristics stratified by Gleason score

\begin{tabular}{|c|c|c|c|c|}
\hline Variable & Total $(n=377)$ & Non-upgraded $(n=209)$ & Upgraded $(n=168)$ & p-value \\
\hline Age (y) & $68(63-72)$ & $68(62-72)$ & $69(64-73)$ & 0.035 \\
\hline PSA (ng/mL) & $7.45(5.41-12.00)$ & $6.80(4.79-10.56)$ & 8.72 (6.09-14.90) & $<0.001$ \\
\hline PV $(m L)$ & $32.0(26.0-40.0)$ & $33(26.0-41.2)$ & $31.1(26.0-38.5)$ & 0.217 \\
\hline $\operatorname{PSAD}\left(\mathrm{ng} / \mathrm{mL}^{2}\right)$ & $0.24(0.16-0.38)$ & $0.20(0.15-0.32)$ & $0.28(0.20-0.46)$ & $<0.001$ \\
\hline Number of positive cores & $3(2-5)$ & $3(2-5)$ & $3(1-6)$ & 0.633 \\
\hline Percent of positive cores & $25.0(16.7-42.9)$ & $25.0(16.7-41.7)$ & $25.0(14.3-49.1)$ & 0.840 \\
\hline Maximum tumor length in a core (mm) & $0.5(0.3-0.8)$ & $0.5(0.3-0.8)$ & $0.6(0.4-0.8)$ & 0.560 \\
\hline Maximum percentage of tumor length in a positive core & $31.7(21.4-50.0)$ & $30.7(20.0-50.0)$ & $33.3(22.2-52.9)$ & 0.262 \\
\hline PI-RADS v2 score & & & & 0.034 \\
\hline 1 & $4(1.1)$ & $3(1.4)$ & $1(0.6)$ & \\
\hline 2 & $2(0.5)$ & $2(1.0)$ & $0(0.0)$ & \\
\hline 3 & $64(17.0)$ & $44(21.1)$ & $20(11.9)$ & \\
\hline 4 & $166(44.0)$ & $93(44.5)$ & $73(43.5)$ & \\
\hline 5 & $141(37.4)$ & $67(32.1)$ & $74(44.0)$ & \\
\hline Number of lesions in mpMRI & & & & 0.425 \\
\hline 1 & $319(84.6)$ & $181(86.6)$ & $138(82.1)$ & \\
\hline 2 & $55(14.6)$ & $27(12.9)$ & $28(16.7)$ & \\
\hline 3 & $3(0.8)$ & $1(0.5)$ & $2(1.2)$ & \\
\hline Clinical T stage & & & & 0.324 \\
\hline $\mathrm{T} 1 \mathrm{~b}-\mathrm{c}$ & $249(66.0)$ & $143(68.4)$ & $106(63.1)$ & \\
\hline $\mathrm{T} 2$ & $82(21.8)$ & $44(21.1)$ & $38(22.6)$ & \\
\hline T3a-b & $46(12.2)$ & $22(10.5)$ & $24(14.3)$ & \\
\hline
\end{tabular}

Values are presented as median (interquartile range) or number (\%).

PSA, prostate-specific antigen; PV, prostate volume; PSAD, PSA density; PI-RADS v2, Prostate Imaging-Reporting and Data System version 2; mpMRI, multiparametric magnetic resonance imaging.

Table 2. Uni- and multivariable logistic regression of clinicopathologic parameters on upgrading

\begin{tabular}{|c|c|c|c|c|}
\hline \multirow{2}{*}{ Variable } & \multicolumn{2}{|c|}{ Univariate } & \multicolumn{2}{|c|}{ Multivariate } \\
\hline & OR $(95 \% \mathrm{Cl})$ & p-value & OR $(95 \% \mathrm{Cl})$ & p-value \\
\hline Age (y) ( $\geq 65$ vs. <65) & $1.627(1.040-2.544)$ & 0.032 & $1.594(1.003-2.532)$ & 0.049 \\
\hline PSA (ng/mL) ( $\geq 7.7$ vs. <7.7) & $2.062(1.365-3.117)$ & $<0.001$ & $1.631(1.032-2.579)$ & 0.036 \\
\hline $\mathrm{PV}(\mathrm{mL})$ & $0.994(0.981-1.008)$ & 0.414 & & \\
\hline $\operatorname{PSAD}\left(\mathrm{ng} / \mathrm{mL}^{2}\right)(\geq 0.475$ vs. $<0.475)$ & $2.707(1.522-4.814)$ & $<0.001$ & $2.034(1.076-3.845)$ & 0.029 \\
\hline Number of positive cores & $1.003(0.925-1.088)$ & 0.937 & & \\
\hline Percent of positive cores & $1.004(0.994-1.015)$ & 0.411 & & \\
\hline Maximum tumor length in a core & $1.191(0.684-2.074)$ & 0.536 & & \\
\hline Maximum percentage of tumor length in a positive core & $1.005(0.996-1.014)$ & 0.267 & & \\
\hline PI-RADS v2 score (4-5 vs. $1-3$ ) & $2.144(1.227-3.746)$ & 0.007 & $1.998(1.130-3.534)$ & 0.017 \\
\hline Number of lesions in mpMRI & $1.404(0.834-2.365)$ & 0.202 & & \\
\hline \multicolumn{5}{|l|}{ Clinical T stage } \\
\hline $\mathrm{T} 1 \mathrm{~b}-\mathrm{c}$ & Reference & & & \\
\hline $\mathrm{T} 2$ & $0.679(0.362-1.277)$ & 0.230 & & \\
\hline T3a-b & $0.792(0.382-1.632)$ & 0.527 & & \\
\hline
\end{tabular}

OR, odd ratio; Cl, confidence interval; PSA, prostate-specific antigen; PV, prostate volume; PSAD, PSA density; PI-RADS v2, Prostate ImagingReporting and Data System version 2; mpMRI, multiparametric magnetic resonance imaging.

However, D'Amico et al. [13] reported that 40\% of the males with low-grade disease at biopsy were found to have high grade disease at prostatectomy and other studies showed that a substantial percentage of patients with GS $3+4$ were upgraded [10,14]. Additionally, GS $\geq 4+3$ disease that has a significantly poor prognosis compared to GS $3+4$ disease 
Table 3. Probability of Gleason upgrading in patients presenting with risk factors $(n=377)$

\begin{tabular}{cccc}
\hline $\begin{array}{c}\text { Number of } \\
\text { risk factors }\end{array}$ & $\mathbf{n}(\%)$ & $\begin{array}{c}\text { Probability of GS } \\
\text { upgrading }\end{array}$ & p-value \\
\hline 0 & $12(3.2)$ & $0 \%$ & $<0.001$ \\
1 & $88(23.3)$ & $36.4 \%$ & \\
2 & $141(37.4)$ & $36.9 \%$ & \\
3 & $101(26.8)$ & $60.4 \%$ & \\
4 & $35(9.3)$ & $65.7 \%$ & \\
\hline
\end{tabular}

GS, Gleason score.

$[15,16]$, needs an active treatment rather than AS. Ploussard et al. [17] reported that the rate of GS upgrading from GS $3+4$ to pathologic GS $\geq 4+3$ was $25.3 \%$. Their study solely enrolled patients diagnosed with biopsy GS $3+4$ and clinical T1-2 stage, whereas our study included all patients with clinical T1-3 stage. As a predisposing risk factor in a few studies, the impact of clinical stage on GS upgrading was taken into account. Similarly, Yang et al. [10] demonstrated that $30.3 \%$ of patients were upgraded when more than 10,000 males with GS 3+4 PCa were analyzed. The subjects in that study fulfilled the NCCN categorization of favorable IR PCa (PSA $<10 \mathrm{ng} / \mathrm{mL}$, cT1c-2a, and positive biopsy score $<50 \%$ ) [10]. Recently, numerous studies reported that PSA level and number of positive biopsy cores were considered significant predictors for pathologic GS upgrading $[10,14,17,18]$. Therefore, the marked differences in the percentage of GS-upgraded patients between their study and ours can be contributed to these eligibility criteria.

As previously mentioned, high-grade $\mathrm{PCa}$ is associated with adverse pathology and an increased risk of PCa-related death $[19,20]$. Consequently, the prediction of GS upgrading plays an important role when considering AS, especially in GS $3+4$ patients. In our study, we found that age, PSA level, PSAD, and PI-RADS v2 score were highly associated with GS upgrading. Ploussard et al. [17] reported that PSA, PSAD, clinical stage, and $>2$ positive cores were significant predictors. Similarly, Yang et al. [10] found the predictors, including age, higher PSA value, clinical stage cT2c versus cTlc, and the percentage of positive cores on multivariable analysis. However, Morlacco et al. [14] showed that only age, PSAD, and the percentage of surface involvement, not clinical stage, were associated with GS upgrading. These discrepancies were probably caused by the diverse population in their study with various selection criteria, such as restricted PSA level, clinical stage, and the number of positive cores.

Notably, age is an independent predictor of GS upgrading confirmed by previous studies of low- and intermediaterisk PCa patients [10,21]. Consistent with our results, Busch et al. [22] reported that pathological upgrading was significantly higher among older ( $\geq 65$ years) versus younger $(<65$ years). With increasing age, males are significantly more likely to have poor prognosis prostate cancer. A possible explanation is the growing tendency to use hormone therapy and watchful waiting/AS among elderly males and less local therapy, particular RP, compared to younger males [23]. A high proportion of young males with low-risk cancer enrolled in AS subsequently receive curative treatment. Alternatively, a large proportion of old males with more aggressive cancer receive watchful waiting than younger males with less aggressive cancer [24]. Additionally, more aggressive prostate cancers possibly arise during andropause when the plasma levels of free testosterone may be below the lower limit of normal. Prior studies found that highgrade prostate cancer was associated with low serum testosterone levels [25,26]. Although the precise mechanism of this phenomenon is incompletely understood, it might be related to the influence of aging on tumor biology, leading to more aggressive differentiation. In general, advanced age should be taken into consideration when discussing treatment for elderly males who have a higher risk of death from competing causes and more comorbidities.

Similar to the aforementioned studies, we found that PSA level was a consistent predictor for GS upgrading after surgery. This outcome supports PSA as a powerful prognostic factor in PCa. Interestingly, prostate volume did not show significant value in predicting GS upgrading, but PSAD was highly associated with GS upgrading in our study. Recent studies also proved that PSAD was an independent predictor of upgrading [14,17], suggesting a critical role in the GS upgrading predictive model.

Recently, mpMRI has become a promising tool in diagnosis and staging of PCa. Many studies confirmed the value of MRI within AS, which has a high specificity for low-risk PCa but in none of studies was MRI used as an indication for treatment [27]. Park et al. [28] revealed that PI-RADS v2 helped preoperatively predict clinically significant cancer, such as GS $\geq 7$, tumor volume $\geq 0.5 \mathrm{~cm}^{2}$, and positive extracapsular extension or seminal vesicle invasion. Song et al. [29] showed that the combination of MRI and clinical parameters had a significantly higher accuracy than clinical variables in GS 6 patients. Our study indicated that PI-RADS v2 score $\geq 4$ was positively associated with an increased risk of upgrading in GS $3+4$ cancer, proposing a powerful predictor to properly evaluate the GS upgrading probability of GS $3+4$ PCa patients.

In recent years, much of research on the value of predictive factors has focused on the relationship between GS 
upgrading and clinical parameters. The important question that emerges is how we can apply these results in daily challenging situation to determine risk classification and counsel patients regarding treatment decisions and disease prognosis. Ploussard et al. [17] assessed three factors including: PSAD $>0.15 \mathrm{ng} / \mathrm{mL}^{2},>2$ positive cores, and clinical stage $\mathrm{T} 2$, and the rate of GS upgrading was $12 \%$ when any factors were present and up to $30.9 \%$ when all three factors were involved. In our study, we structured a simple predictive model with four factors: age $\geq 65, \mathrm{PSA} \geq 7.7 \mathrm{ng} / \mathrm{mL}, \mathrm{PSAD} \geq 0.475 \mathrm{ng} / \mathrm{mL}^{2}$, and PI-RADS v2 score $\geq 4$. The more risk factors the GS $3+4 \mathrm{PCa}$ patient has, the higher GS upgrading probability is. Indeed, the rate of GS upgrading ranged from $36.4 \%$ to $65.7 \%$ when one to 4 factors were present.

Our study was not devoid of limitations. First, our findings came from a retrospective study at a single institution. The retrospective nature and the lack of a single experienced pathologist reexamination of all cases are potential limitations. Second, the patients were selected for RP and may be not representative of all patients with GS $3+4$ who underwent radiotherapy, watchful waiting or another therapy and that introduced a selection bias in study. Finally, we did not analyze some characteristics of biopsy sample, such as percentage of GS 4 and tertiary GS 5, which might relate to poor PCa prognoses. Further studies with a larger data set analyzing adequate information on biopsy samples are necessary to estimate the relationship between variables and adverse pathology.

\section{CONCLUSIONS}

Approximately half of the patients with biopsy GS 3+4 cancer was upgraded after RP. The risk of GS upgrading included advanced age, high PSA, high PSAD, and PI-RADS score $4-5$ identify patients as poor candidates for AS. Our results suggest a useful tool that can be applied easily in clinical circumstances to determine the risk classification and recommend patients for reasonable treatments.

\section{CONFLICTS OF INTEREST}

The authors have nothing to disclose.

\section{AUTHORS' CONTRIBUTIONS}

Sang Eun Lee had full access to all the data in the study and takes responsibility for the integrity of the data and the accuracy of the data analysis. Study concept and design: Duc Minh Pham, Jung Kwon Kim, and Sang Eun Lee.
Acquisition of data: Duc Minh Pham, Sangchul Lee, Sung Kyu Hong, and Seok-Soo Byun. Analysis and interpretation of data: Duc Minh Pham. Drafting of the manuscript: Duc Minh Pham and Jung Kwon Kim. Critical revision of the manuscript for important intellectual content: all authors. Statistical analysis: Duc Minh Pham. Supervision: Sang Eun Lee. Other: none.

\section{REFERENCES}

1. Siegel RL, Miller KD, Jemal A. Cancer statistics, 2019. CA Cancer J Clin 2019;69:7-34.

2. Dahabreh IJ, Chung M, Balk EM, Yu WW, Mathew P, Lau J, et al. Active surveillance in men with localized prostate cancer: a systematic review. Ann Intern Med 2012;156:582-90.

3. Raldow AC, Zhang D, Chen $\mathrm{MH}$, Braccioforte $\mathrm{MH}$, Moran BJ, D'Amico AV. Risk group and death from prostate cancer: implications for active surveillance in men with favorable intermediate-risk prostate cancer. JAMA Oncol 2015;1:334-40.

4. Cooperberg MR, Cowan JE, Hilton JF, Reese AC, Zaid HB, Porten SP, et al. Outcomes of active surveillance for men with intermediate-risk prostate cancer. J Clin Oncol 2011;29:228-34.

5. Hamdy FC, Donovan JL, Lane JA, Mason M, Metcalfe C, Holding $\mathrm{P}$, et al. 10-year outcomes after monitoring, surgery, or radiotherapy for localized prostate cancer. N Engl J Med 2016;375:1415-24.

6. NCCN Clinical Practice Guidelines in Oncology (NCCN Guidelines $^{\circledR}$ ). Prostate Cancer, Version 4. 2019 [Internet]. National Comprehensive Cancer Network; 2019. Available from: https://www.nccn.org/professionals/physician_gls/pdf/prostate.pdf.

7. Chen RC, Rumble RB, Loblaw DA, Finelli A, Ehdaie B, Cooperberg MR, et al. Active Surveillance for the Management of Localized Prostate Cancer (Cancer Care Ontario guideline): American Society of Clinical Oncology clinical practice guideline endorsement. J Clin Oncol 2016;34:2182-90.

8. Jain S, Loblaw A, Vesprini D, Zhang L, Kattan MW, Mamedov A, et al. Gleason upgrading with time in a large prostate cancer active surveillance cohort. J Urol 2015;194:79-84.

9. Zumsteg ZS, Spratt DE, Pei I, Zhang Z, Yamada Y, Kollmeier M, et al. A new risk classification system for therapeutic decision making with intermediate-risk prostate cancer patients undergoing dose-escalated external-beam radiation therapy. Eur Urol 2013;64:895-902.

10. Yang DD, Mahal BA, Muralidhar V, Nezolosky MD, Vastola ME, Labe SA, et al. Risk of upgrading and upstaging among 10000 patients with Gleason 3+4 favorable intermediate-risk prostate cancer. Eur Urol Focus 2019;5:69-76.

11. Klotz L, Zhang L, Lam A, Nam R, Mamedov A, Loblaw A. 
Clinical results of long-term follow-up of a large, active surveillance cohort with localized prostate cancer. J Clin Oncol 2010;28:126-31.

12. van den Bergh RC, Roemeling S, Roobol MJ, Aus G, Hugosson J, Rannikko AS, et al. Gleason score 7 screen-detected prostate cancers initially managed expectantly: outcomes in 50 men. BJU Int 2009;103:1472-7.

13. D'Amico AV, Renshaw AA, Arsenault L, Schultz D, Richie JP. Clinical predictors of upgrading to Gleason grade 4 or 5 disease at radical prostatectomy: potential implications for patient selection for radiation and androgen suppression therapy. Int J Radiat Oncol Biol Phys 1999;45:841-6.

14. Morlacco A, Cheville JC, Rangel LJ, Gearman DJ, Karnes RJ. Adverse disease features in Gleason score $3+4$ "favorable intermediate-risk" prostate cancer: implications for active surveillance. Eur Urol 2017;72:442-7.

15. Chan TY, Partin AW, Walsh PC, Epstein JI. Prognostic significance of Gleason score 3+4 versus Gleason score 4+3 tumor at radical prostatectomy. Urology 2000;56:823-7.

16. Park J, Yoo S, Cho MC, Cho MH, Jeong CW, Ku JH, et al. The impact of pathologic upgrading of Gleason score 7 prostate cancer on the risk of the biochemical recurrence after radical prostatectomy. Biomed Res Int 2018;2018:4510149.

17. Ploussard G, Isbarn H, Briganti A, Sooriakumaran P, Surcel CI, Salomon L, et al. Can we expand active surveillance criteria to include biopsy Gleason 3+4 prostate cancer? A multi-institutional study of 2,323 patients. Urol Oncol 2015;33:71.e1-9.

18. Hong SK, Han BK, Lee ST, Kim SS, Min KE, Jeong SJ, et al. Prediction of Gleason score upgrading in low-risk prostate cancers diagnosed via multi ( $>$ or $=12$ )-core prostate biopsy. World J Urol 2009;27:271-6.

19. Albertsen PC, Hanley JA, Fine J. 20-year outcomes following conservative management of clinically localized prostate cancer. JAMA 2005;293:2095-101.

20. Patel HD, Tosoian JJ, Carter HB, Epstein JI. Adverse pathologic findings for men electing immediate radical prostatectomy: defining a favorable intermediate-risk group. JAMA Oncol 2018;4:89-92.

21. Winters BR, Wright JL, Holt SK, Lin DW, Ellis WJ, Dalkin BL, et al. Extreme Gleason upgrading from biopsy to radical prostatectomy: a population-based analysis. Urology 2016;96:14855.

22. Busch J, Magheli A, Leva N, Ferrari M, Kramer J, Klopf C, et al. Higher rates of upgrading and upstaging in older patients undergoing radical prostatectomy and qualifying for active surveillance. BJU Int 2014;114:517-21.

23. Bechis SK, Carroll PR, Cooperberg MR. Impact of age at diagnosis on prostate cancer treatment and survival. J Clin Oncol 2011;29:235-41.

24. Loeb S, Berglund A, Stattin P. Population based study of use and determinants of active surveillance and watchful waiting for low and intermediate risk prostate cancer. J Urol 2013;190:1742-9.

25. Hoffman MA, DeWolf WC, Morgentaler A. Is low serum free testosterone a marker for high grade prostate cancer? J Urol 2000;163:824-7.

26. Schatzl G, Madersbacher S, Thurridl T, Waldmüller J, Kramer G, Haitel A, et al. High-grade prostate cancer is associated with low serum testosterone levels. Prostate 2001;47:52-8.

27. van den Bergh RC, Ahmed HU, Bangma CH, Cooperberg MR, Villers A, Parker CC. Novel tools to improve patient selection and monitoring on active surveillance for low-risk prostate cancer: a systematic review. Eur Urol 2014;65:1023-31.

28. Park SY, Jung DC, Oh YT, Cho NH, Choi YD, Rha KH, et al. Prostate cancer: PI-RADS version 2 helps preoperatively predict clinically significant cancers. Radiology 2016;280:108-16.

29. Song W, Bang SH, Jeon HG, Jeong BC, Seo SI, Jeon SS, et al. Role of PI-RADS version 2 for prediction of upgrading in biopsy-proven prostate cancer with Gleason score 6. Clin Genitourin Cancer 2018;16:281-7. 